\title{
EPILEPSY AND AFFECTIVE DISORDERS: DEPRESSION AS A SUICIDE RISK (REVIEW ARTICLE)
}

\author{
Gulnoza Ruzieva, Komila Porsokhonova, Bekhzod \\ Abdullaev \\ Department of Psychiatry, Tashkent Medical Academy, \\ Tashkent, Uzbekistan
}

Contact Details:

author.uzb@mail.ru

Farobi 2, Almazar District

Tashkent, Uzbekistan 100109

\begin{abstract}
Affective disorders are perhaps the main practical importance among the variety of mental disorders in patients with epilepsy, defining the range of diagnostic and differentialdiagnostic problems, treatment and rehabilitation tactics. Incidentally arising in patients with epilepsy' mood disorders attracted the attention of many researchers. Mood disorders are considered as diagnostic feature for the recognition of genuine, "true" epilepsy, with the possible absence of other manifestations of the disease and indicates that the leading place in the structure of mental disorders in epilepsy is affective disorder observed in the onset of the disease and the disease process.

\section{Introduction}

Currently, the goal of the doctor treating a patient with epilepsy is not only preventing attacks, but also help the patient in optimizing his quality of life. Meanwhile, the quality of life of
\end{abstract}


patient with epilepsy not only affects the presence or absence of drug-free remission but also its psychosocial adaptation to his illness and is associated with the presence or lack of affective disorders. It is known that the risk of suicide is 5 times higher in patients with epilepsy and 25 times higher in temporal lobe epilepsy and complex partial seizures than in the General population.

The main factors that increase the risk of suicidal behavior in epilepsy, consider concomitant affective disorders (depression) and psychosis. Suicidal thoughts, suicide attempts and completed suicide represent different states within the continuum, where suicidal thoughts can be placed on one end of the spectrum, to attempted suicide in the center and completed suicide at the other end of the spectrum. Many authors indicate a relationship between epilepsy and increased risk of suicide. Suicide is an important cause of death in patients with epilepsy, the frequency and significance of which is often underestimated. The relationship between epilepsy and suicide is complex, multifactorial and bidirectional. The reason may serve as features of the disease - epilepsy (depending on the form of epilepsy, type of seizure, location of brain damage), and limitations encountered by people with epilepsy in society (social stigma or "stigma of epilepsy"); also, we cannot exclude a causal role of some antiepileptic drugs (AEDs) [1].

\section{Epilepsy and suicide}

The frequency of suicidal phenomena significantly higher in patients with epilepsy than in general population and reaches 8-12\%, compared to 1.1-1.2 percent in general population and even higher (about 25 times) in temporal lobe epilepsy and complex partial seizures $[1,2]$. Any case of completed suicide is always the result of a complex set of factors. In turn, some of these factors are interrelated. The relationship between epilepsy and increased risk of suicide may influence psychiatric, demographic (including sex-linked) and socio-economic factors. However, even adjusted for these factors, the risk of suicidal behavior in people with epilepsy is much higher than in general population. Risk factors, of course, is related to psychiatric disorders: affective disorders. 


\section{Epilepsy and depression}

According to most authors $[1,2,3]$, the main reason for the development of affective disorders (depression) or psychosis in a patient with epilepsy that may be caused by disease, and AEDs. Most important, according to many authors, is the increased frequency of accompanying mental disorders, particularly affective and anxiety disorders. The risk of suicide with these disorders varies, but not installed. However, it is considered that special importance are mood disorders (depression) and epileptic psychoses, in which the possible suicidal thoughts and behavior. Note that in many cases mental disorders in epilepsy not diagnosed in a timely manner. In the study, Christensen J. et al. (2007) the risk of suicide was particularly high in patients with concomitant psychiatric disease in the anamnesis. Kwon O.Y., Park S.P. (2013) examined 568 patients with epilepsy with neuropsychological questionnaire that includes metrics to assess symptoms of depression, anxiety, social stigma, suicidal ideation and quality of life. In $30.5 \%$ of patients were identified with emotional disorders. The frequency of depression and anxiety is $27.8 \%$ and $15.3 \%$, respectively, and was significantly higher than in healthy persons of the control group. The frequency of patients feeling "stigma of epilepsy" and frequency of suicidal thoughts was higher in patients with epilepsy and affective disorders compared with those without affective disorders.

H. Hecimovic et al. (2012) prospectively examined 193 consecutively recruited adult patients with epilepsy to assess the level of depression and suicidal thoughts. The proportion of patients who have had suicidal thoughts for the last 2 weeks was $11.9 \%$. Although these analyzed factors, such as the toxic effects of therapy, health-related quality of life and the indicator of the Beck depression scale was associated with suicidal thoughts when conducting bivariate analysis, only one indicator - a score on a Beck depression inventory - maintained statistical significance when applying logistic regression. However, 1/4 of patients with suicidal thoughts significant symptoms of depression were absent [4].

The study Buljan R., Santic A. M. (2011) were the reasons of suicidal behaviour of patients with epilepsy receiving 
treatment in a hospital: $14.6 \%$ of epilepsy patients have made suicide attempts. Unfavorable atmosphere in the family and related psychiatric pathology played a significant role in the formation of suicidal behavior. In this study, the factors associated with the disease, did not play a statistically significant role in establishing the link with suicidal behavior [5].

The relationship between epilepsy and depression is bidirectional - not only epilepsy increases the risk of depression and suicide, but also depression with suicidal phenomena may increase the risk of epileptic seizures.

It is important that the relationship of epilepsy and affective disorders (that increase the risk of suicide) are often underestimated. Psychological research has identified in patients with epilepsy (31 patients) with long-lasting symptoms of mental disorders - $21(91.4 \%)$ case - suicide attempts, however, only 5 (16\%) patients were examined by a psychiatrist, and only 3 of them received treatment. The authors emphasize that the known fact of the frequent combination of epilepsy and affective disorders are often underestimated, and affective disorders often are not detected in a timely manner. At the same time overdose of AEDs can lead to death, so it is necessary to assess the risk of suicide among patients with epilepsy [6].

A number of scholars have obtained data indicating a reduction in the frequency of seizures in patients before the manifestation of depression. Mendez et al. found that patients with epilepsy associated with depression, were recorded fewer generalized seizures than in patients that do not have affective disorders. The authors suggested that non-reactive depression can be a consequence of suppression of generalized epileptic activity from the epileptogenic focus [8].

\section{How can we identify depression in epilepsy?}

Depression in patients with epilepsy can often manifest symptoms that can be viewed as side effects of antiepileptic drugs and as a manifestation of epilepsy per se. Such a disorienting physician complaints can include trouble to sleep, changes in appetite, decreased libido, braking or excessive anxiety, difficulties with concentration and behavioral disorders. 
Classic depressive symptoms rarely occur in patients with epilepsy. According to a study by Mendez et al., in 50\% of cases in patients with epilepsy depression is manifested atypical.

Blumer et al. described a pleomorphic affective disorder epilepsy, characterized by 8 key symptoms such as: labile depressive symptoms (depressive behaviour, asthenia, insomnia, pain), labile affective symptoms (fear, anxiety) and supposedly "specific" symptoms (irritability, euphoric mood) [7].

Kanner et al. prefers the term, "disorder, like dysthymia, epilepsy" and says the presence of this disorder in $70 \%$ of patients with epilepsy requiring treatment. The atypical symptoms of depression in patients with epilepsy leads to a low detection rate [5].

It is important to remember that patients with epilepsy suffers from a number parietal symptoms lasting for hours or days. Perhaps these parietal violations to some extent are the cause of atypical manifestations of depression in epilepsy. Parietal symptoms usually occur dysphoria appearing a few days or hours before the attack and continuing for several days thereafter.

The connection between the attack and postictal disorders can be subtle, as the "lucid interval" between them can last from 1 to 5 days.

Depression and anxiety disorders (characteristic, according to various estimates, $25-50 \%$ of patients with epilepsy) have a significant impact on the quality of life of patients with epilepsy, leading to growth in the number of suicides. According to various estimates, from 5 to $14 \%$ of patients with epilepsy commit suicide or attempt suicide (compared to $1,1-1,2 \%$ in general population).

Identified a number of risk factors for suicidal behavior in patients with epilepsy. In a number of studies have shown a connection to increased risk of suicide after temporal lobectomy and left-sided resection with worsening of seizures and rightsided temporal resection, which has led to the emergence of psychotic symptoms and worsening of seizures. Risk factors for completed suicide include: a history of inflicting deliberate selfharm, cases of suicide in the family, serious stressful situations, 
decrease mood, stigma and psychiatric disorders, especially such as alcoholism or the abuse of prescription substances as well as interictally depression, psychosis and personality change.

Moreover, Blumer D. et al. (1991) introduced the concept of "interictal dysphoric disorder", which can be a predictor of development interictal psychosis, and suicide [1].

\section{Interictal depression}

It is a mood disorder that occurs in the period between attacks has a different (often-short) duration, and a tendency to self-limitation.

Blumer, 1998 noticed that this particular mood disorder is inherent in patients with refractory epilepsy, especially in the localization of epileptic focus in the temporal lobe. As a rule, interictally mood disorders occur years later (two or more) after the debut of epilepsy. Interictal dysphoric symptoms are presented in various combinations and typically last a relatively short (from several hours to two or three days). Blumer and coauthors identified eight affective-somatoform symptom dysphoria.

Most researchers believe that diagnosis interictal dysphoric disorder enough the presence of three symptoms. Interestingly, the usual premenstrual dysphoric disorder in symptomology identical interictal dysphoric disorder. In women suffering from epilepsy and experiencing interictal dysphoric disorder psychopathology, symptoms intensify and expand the adult period. Interictal dysphoric disorder is considered as a risk factor for sudden suicide attempts and interictal psychosis. Anxiety often accompanies depression in primary mental disorders. At least half of the patients with depression have anxiety symptoms. The presence of anxiety symptoms may also significantly degrade the quality of life of patients and increase the risk of suicide. Kanner A.M. (2004) with co-authors utilized a structured clinical questionnaire for the diagnosis of psychiatric disorders according to DSM-IV, found that symptoms in one third of patients with epilepsy corresponds to the major depressive episode, dysthymia, anxiety disorder, mixed anxiety-depressive disorder $[5,6]$. 
Depression is extremely violates the patient's quality of life. Our study showed that the overall quality of life of patients with epilepsy and comorbid depression significantly reduced.

The study of Johnson et al., (2004) also carried out in patients with focal epilepsy using a regression analysis showed that the contribution of depression to the decline in the quality of life of patients with epilepsy is $35 \%$, and factors associated with epilepsy less than 20\%. Moreover, the significant impact of depression on quality of life persisted after achieving seizure control or reducing frequency and severity of seizures, as well as leveling other negative psychosocial factors.

Our data also indicate that affective disorders are frequent concomitant symptoms in epilepsy: in 22 patients, including 13 women and 9 men with epilepsy in the anamnesis pointed to suicide attempts. After a psychiatric evaluation revealed that: 14 patients suffering depressive episode, 3 patients agitated depression, 6 bipolar patients, and 2 patients with anxiety disorder. Causes of suicide attempts, patients identified the following factors: epileptic stigma (patients with depressive episodes) social maladjustment that is, divorces in the family because of the diagnosis, inability to create a family, find a job (patients with anxiety disorders, bipolar disorder).

On the basis of study of archival data, medical history, clinical observations, and data of instrumental studies of the etiological factors of depression in epilepsy multifactorial in nature. Many factors affect the formation of depression can be divided into four categories:

- Iatrogenic factors

- Social stigma of epilepsy

- Genetic factors

- Associated with epileptic disorders neurophysiological and neurochemical processes.

Therefore, a fatal consequence of depression in epilepsy is suicide. In addition to the typical consequences (reduced quality of life, social functioning, suicide) depression in patients with epilepsy, can cause aggravation seizures or reduced seizure control. The worsening course of epilepsy only partially associated with depression induced by incompletely. On the 
contrary, the correction of depression contributes to seizure control.

\section{Findings}

Thus, literature data and our own data allow us to conclude that:

1) Depression is a major factor that hinders the quality of life of epilepsy patients and its pathogenic effect higher than the effect of the severity of the disease (frequency, severity of seizures, response to treatment, and duration of illness).

2) The basis of suicidal behavior in all cases are depression, dysphoria and anxiety.

3) $70-80 \%$ of patients with epilepsy suffer from chronic depression, or a depressive episode of varying duration occurring at least once during the disease.

4) Depression in epilepsy have a multifactorial etiology.

5) Experts in the field of epileptology should actively identify in their patients the presence of suicidal thoughts and should be aware of the existence of the risk of suicide in patients with epilepsy.

\section{References:}

1. Burd S. G., Reader F. K., Badalyan O. L., Avakyan G. G., Chukanov S. A. Depression and epilepsy: two sides of the same coin // Rus. Med. Jour. (Neuroscience). - 2008. - T. 16. - No. 12. - S. 1-5.

2. Kalinin V. V. Epilepsy as a neuropsychiatric problem // Psychiatry and pharmacotherapy. - 2004. -Vol 06 (1).

3. Kalinin VV, Polyanskiy DA. Risk factors for suicidal behavior in patients with epilepsy // Journal Neurol Psychiatry - 2003. Vol. 103, No. 3. - Pp. 18-21.

4. Pompili $\mathrm{M}$ et al. Depression, hopelessness and suicide risk among patients suffering from epilepsy. Ann 1st Super Sanita 2007 Vol. 43, No. 4; 425-429. 5. Kanner A.M. Depression and Epilepsy: A New Perspective on Two Closely Related Disorders. Epilepsy Currents, Vol. 6, No 5 (September/October) 2006

pp.

141-146.

7. Kimiskidis Vasilios $\mathrm{K}$ et al. Depression and anxiety in epilepsy: the association with demographic and seizure-related variables. Annals of General Psychiatry 2007; 6:28. 
8. Lambert M.V. and Robertson M.M. Depression in Epilepsy: Etiology, Phenomenology and Treatment. Epilepsia 1999, Vol. 40, Suppl. 10: S21-47. 9. Loring D.W. and Meador K.J. Cognitive and Behavioral Effects of Epilepsy Treatment. Epilepsia 2001, 42 (Suppl. 8): 2432.

10. Meador K.J. et al. Cognitive and behavioral effects of lamotrigine and topiramate in healthy volunteers. Neurology 2005; 64; 2108-2114. 